MATHEMATICS OF COMPUTATION

Volume 67, Number 222, April 1998, Pages 541-552

S 0025-5718(98)00942-9

\title{
DERIVATIVE SUPERCONVERGENT POINTS IN FINITE ELEMENT SOLUTIONS OF POISSON'S EQUATION FOR THE SERENDIPITY AND INTERMEDIATE FAMILIES - A THEORETICAL JUSTIFICATION
}

\author{
ZHIMIN ZHANG
}

\begin{abstract}
Finite element derivative superconvergent points for the Poisson equation under local rectangular mesh (in the two dimensional case) and local brick mesh (in the three dimensional situation) are investigated. All superconvergent points for the finite element space of any order that is contained in the tensor-product space and contains the intermediate family can be predicted. In case of the serendipity family, the results are given for finite element spaces of order below 7. Any finite element space that contains the complete polynomial space will have at least all superconvergent points of the related serendipity family.
\end{abstract}

\section{INTRODUCTION}

In the development of the finite element method, researchers have observed that for certain classes of problems, the rate of convergence of the values of the finite element solution and/or its derivatives at some special points exceeds the possible global rate. This phenomenon has been termed "superconvergence" and has been analyzed mathematically because of its practical importance in finite element computations. For the literature, the reader is referred to [7]. Regarding the finite element superconvergent points for the Poisson equation, a result can be found from the early 70's in [3], where the authors proved that element knots are superconvergent points when the tensor product $C^{0}$ elements are used.

The term superconvergence also includes accelerated convergence achieved by means of various recovery (or post-processing) techniques.

Our consideration in this study is the natural derivative superconvergent points when no post-processing is applied. Note that Douglas et al. [3] considered solution superconvergence, not derivative superconvergence.

In the one dimensional setting, it has been known since the late 70's that the Gaussian points, i.e., zeros of Legendre polynomials, are derivative superconvergent points when using $C^{0}$ elements to solve two-point boundary value problems [2]. But, in the higher dimensional setting, the situation is more complicated in that the superconvergent points are very sensitive to the properties of the solutions, the finite element space, and the geometric pattern of the meshes [1].

Received by the editor May 29, 1996.

1991 Mathematics Subject Classification. Primary 65N30.

This work was supported in part by NSF Grants DMS-9626193 and DMS-9622690.

(C)1998 American Mathematical Society 
In this paper, we shall study the derivative superconvergent points for the Poisson equation under local rectangular meshes in both two and three dimensional settings. There are three popular polynomial bases for two dimensional rectangular meshes in engineering practice: (1) Tensor-product spaces; (2) Serendipity families; (3) Intermediate elements. It is well known that for a large class of problems, superconvergent points for the tensor product space lie along Gaussian lines. Superconvergent points for intermediate and serendipity families were reported only recently.

Recently, there were two important works in the field: A purely theoretical investigation by Schatz, Sloan, and Wahlbin [7] and a "computer-based" proof by Babuška, Strouboulis et al. [1]. The authors of [5] showed for the scalar secondorder elliptic problem that if the finite element space is locally symmetric about a point $x_{0}$ with respect to the antipodal map $x \mapsto x_{0}-\left(x-x_{0}\right)$, then derivative superconvergence occurs at $x_{0}$ when the finite element space is piecewise polynomial of odd degree under mild conditions on the behavior of the solution outside a neighborhood of $x_{0}$. The approach in [1] is quite different. The authors claimed that the existence of superconvergent points can be guaranteed within a given tolerance by a numerical algorithm. With this approach, they found, with the aid of the computer, all superconvergent points for the Laplace equation, the Poisson equation, and the linear elasticity equation, on four mesh patterns of triangular elements and the above three families of rectangular elements of degree $n, 1 \leq n \leq 7$. The main assumptions in [1] are: (a) there is no roundoff error; (b) the meshes are locally translation-invariant; (c) the solution is sufficiently smooth locally. The main idea is to majorize the finite element solution error by a polynomial of one degree higher than the finite element space being used. Therefore, the search for superconvergent points is transferred to a search for intersections of some polynomial contours. At this moment, the computer is used to actually locate those intersections.

In the present work, we shall analytically find those intersections which represent superconvergent points for the Poisson equation under local rectangular meshes, and thereby provide a theoretical justification for superconvergence results, in this case, for the computer-based proof. Furthermore, the analytic approach is capable of predicting some parallel results for many other rectangular elements and brick elements (three dimensional rectangular elements) which have not been reported in the literature. Another advantage of this investigation is the elimination of Assumption (a) in the computer-based proof.

\section{Preliminaries}

We shall outline the approach by Babuška et al. [1] in finding superconvergent points via a computer-based proof. Here we follow the description provided by Wahlbin [7] (Chapter 12).

Consider the local superconvergent points of the finite element solution $u_{h}$ to the solution of the Poisson equation $u$ on a master cell $c\left(x^{0}, h\right)=\left\{y:\left|y-x^{0}\right| \leq h\right\}$. Here $|\cdot|$ denote the $L_{\infty}$-norm of vectors. Assume that the $2 h$-periodic extension of the master cell fits two squares $\Omega_{1}=\left\{y:\left|y-x^{0}\right| \leq 2 H\right\}$ and $\Omega_{0}=\left\{y:\left|y-x^{0}\right| \leq 4 H\right\}$ exactly, where $H=h^{\delta}$ with $0<\delta<1$. As the finite element solution of the Poisson equation, $u_{h}$ satisfies

$$
\int_{\Omega_{0}} \nabla\left(u-u_{h}\right) \nabla v=0, \quad \forall v \in S_{h}^{c o m p}\left(\Omega_{0}\right),
$$


where $S_{h}^{c o m p}\left(\Omega_{0}\right)$ is the finite element subspace which has compact support on $\Omega_{0}$. The basic assumption is

$$
\left\|u-u_{h}\right\|_{L_{\infty}\left(\Omega_{0}\right)} \leq C h^{n+1-L},
$$

with $L+\delta<1$.

Remark 2.1. Assumption (2.1) implies that various pollution effects from outside of $\Omega_{0}$ have been properly treated and the error loss is of order $h^{L}$.

Let $Q$ be the $(n+1)$ th order Taylor-expansion of $u$ at $x_{0}$. Then

$$
\|u-Q\|_{W_{\infty}^{s+1}\left(\Omega_{0}\right)} \leq C H^{n+1-s}, \quad-1 \leq s \leq n .
$$

Denote by $I_{h}$ the standard interpolation operator into $S_{h}\left(\Omega_{0}\right)$, and let $\rho=Q-I_{h} Q$. The key observation in [1] is that $\rho$ is $2 h$-periodic. Let $S_{h}^{\pi}\left(c\left(x^{0}, h\right)\right)$ be $2 h$-periodic functions in $S_{h}\left(c\left(x^{0}, h\right)\right)$, and define $P P(\rho)$ by

$$
\int_{c\left(x^{0}, h\right)}(\rho-P P(\rho))=0, \quad \int_{c\left(x^{0}, h\right)} \nabla(\rho-P P(\rho)) \nabla v=0, \quad \forall v \in S_{h}^{\pi}\left(c\left(x^{0}, h\right)\right) .
$$

$P P(\rho)$ is also used to represent its $2 h$-periodic extension. Now let $\psi=\rho-P P(\rho)$, and we have (cf., [1], [7]):

\section{Theorem 2.1.}

$$
\frac{\partial}{\partial x_{i}}\left(u-u_{h}\right)(x)=\frac{\partial \psi}{\partial x_{i}}(x)+R_{i}(x), \quad x \in \Omega_{0}, \quad i=1,2,
$$

with

$$
\left\|R_{i}\right\|_{L_{\infty}\left(\Omega_{1}\right)} \leq C\left(h^{n+\delta}+h^{n+1-L-\delta}\right) .
$$

Remark 2.2. Theorem 2.1 is our starting point. It states that the finite element approximation error in the derivatives can be majorized by $\frac{\partial \psi}{\partial x_{i}}$, since the remainder $R_{i}$ is of order $\min (\delta, 1-L-\delta)$ higher than the global optimal rate. Therefore, derivative superconvergent points are those points where $\frac{\partial \psi}{\partial x_{i}}(x)=0$. Thus, the task of finding superconvergent points is narrowed down to the task of identifying, on the master cell, the critical points of some $2 h$-periodic piecewise monomials of degree $n+1$ not in the local finite element space $S_{h}\left(c\left(x^{0}, h\right)\right)$. This is equivalent to finding the critical points of some $\psi$, periodic monomials of degree $n+1$ on the reference element $\widehat{K}=[-1,1]^{2}$ satisfying $\psi \notin V_{n}(\widehat{K})$, and

$$
\int_{\widehat{K}} \nabla \psi \nabla v=0, \quad \forall v \in V_{n}(\widehat{K}),
$$

where $V_{n}(\widehat{K})$ is the image of $S_{h}\left(c\left(x^{0}, h\right)\right)$ under the change of variables $x_{1}=x_{1}^{0}+h \xi$, $x_{2}=x_{2}^{0}+h \eta$. In [1], the job of locating critical points of some periodic polynomials is done by the computer, so it is called "computer-based proof". In the present study, we shall find them analytically by characterizing the space of periodic polynomials.

Remark 2.3. Theorem 2.1 can be generalized to the three dimensional setting (cf., $[1])$. 


\section{Characteristic OF PERIOdic POlynomials}

We shall characterize the space of periodic polynomials of degree not greater than $n$ on $\widehat{K}=[-1,1]^{2}$, denoted as $P P_{n}(\widehat{K})$.

Define $P(\widehat{K})$, the set of periodic functions on $\widehat{K}$, i.e., $f(\xi, 1)=f(\xi,-1), f(1, \eta)=$ $f(-1, \eta)$ for $f \in P(\widehat{K})$. Let $P_{n}(\widehat{K})$ denote the space of polynomials of degree not greater than $n$ on $\widehat{K}$. Then

$$
P P_{n}(\widehat{K})=P_{n}(\widehat{K}) \cap P(\widehat{K}) .
$$

Observe that for any polynomial $p$ of a single variable,

$$
P(\widehat{K}) \cap \operatorname{Span}\{p(\xi) \eta, \xi p(\eta)\}=\{0\} .
$$

Therefore,

$$
P P_{n}(\widehat{K})=S_{n}(\widehat{K}) \cap P(\widehat{K}),
$$

where

$$
S_{n}(\widehat{K})=P_{n}(\widehat{K}) \cup \operatorname{Span}\left\{\xi^{n} \eta, \xi \eta^{n}\right\} .
$$

Note that $S_{n}(\widehat{K})$ is the serendipity family which contains 4 nodal shape functions, $4(n-1)$ side modes, and $(n-3)(n-2) / 2$ internal modes (cf., Section 6.1 in [6]).

Dimension of $P P_{n}(\widehat{K})$ can be decided by deleting from $S_{n}(\widehat{K})$ : (a) 3 nodal freedoms (one node decides the other three by periodicity) and, (b) $2(n-1)$ side modes (two sides decide the remaining two by periodicity), since all internal modes of $S_{n}(\widehat{K})$ are automatically periodic. Hence,

$$
\begin{aligned}
\operatorname{dim} P P_{n}(\widehat{K}) & =\operatorname{dim} S_{n}(\widehat{K})-2(n-1)-3 \\
& =\frac{(n+1)(n+2)}{2}+2-2(n-1)-3=\frac{(n-1) n}{2}+2, \quad n \geq 2 .
\end{aligned}
$$

Letting $p_{k}$ denote the Legendre polynomial of degree $k(\geq 0)$, we define

$$
\phi_{k+1}(\xi)=\int_{-1}^{\xi} p_{k}(t) d t, \quad k \geq 1,
$$

$$
T_{n}(\widehat{K})=\operatorname{Span}\left\{1, \phi_{k}(\xi), \phi_{k}(\eta), k=2,3, \ldots, n ; \phi_{i}(\xi) \phi_{j}(\eta), i+j \leq n, i, j \geq 2\right\} .
$$

Using the recurrence relation (page 47 in [4]),

$$
p_{k+1}^{\prime}(t)-p_{k-1}^{\prime}(t)=(2 k+1) p_{k}(t), \quad k \geq 1,
$$

we see that,

$$
\phi_{k+1}(\xi)=\frac{1}{2 k+1}\left(p_{k+1}(\xi)-p_{k-1}(\xi)\right), \quad k \geq 1 .
$$

\section{Theorem 3.1.}

$$
P P_{n}(\widehat{K})=T_{n}(\widehat{K})
$$

Proof. Obviously $T_{n}(\widehat{K}) \subset P_{n}(\widehat{K})$. Observe that $\phi_{j}(-1)=\phi_{j}(1)=0(j \geq 2)$. Hence $T_{n}(\widehat{K}) \subset P(\widehat{K})$ and therefore $T_{n}(\widehat{K}) \subset P_{n}(\widehat{K}) \cap P(\widehat{K})=P P_{n}(\widehat{K})$. Notice 
that all polynomials in (3.1) are linearly independent. Therefore, in order to prove $T_{n}(\widehat{K})=P P_{n}(\widehat{K})$, we only need to show that $\operatorname{dim} T_{n}(\widehat{K})=\operatorname{dim} P P_{n}(\widehat{K})$. Indeed,

$$
\begin{aligned}
\operatorname{dim} T_{n}(\widehat{K}) & =1+2(n-1)+1+2+\cdots+n-3 \\
& =\frac{(n-1) n}{2}+2=\operatorname{dim} P P_{n}(\widehat{K}) . \quad
\end{aligned}
$$

Theorem 3.1 characterizes the space of periodic polynomials (on $\widehat{K}$ ). Further, we consider the orthogonal decomposition of $P P_{n}(\widehat{K})$ under the Laplace operator. Towards this end, we define

$$
\Psi_{n}(\widehat{K})=\left\{u \in P P_{n}(\widehat{K}) \mid \quad \int_{\widehat{K}} \nabla u \nabla v=0 \quad \forall v \in P P_{n-1}(\widehat{K})\right\} .
$$

Then by the Gram-Schmidt process, we can decompose $P P_{n}(\widehat{K})$ into

$$
P P_{n}(\widehat{K})=P P_{0}(\widehat{K}) \oplus \Psi_{2}(\widehat{K}) \oplus \cdots \oplus \Psi_{n-1}(\widehat{K}) \oplus \Psi_{n}(\widehat{K}) .
$$

Note that $P P_{0}(\widehat{K})=\operatorname{Span}\{1\}$ and $\Psi_{1}(\widehat{K})=\{0\}$.

\section{Derivative Superconvergent Points For ReCtangular ElEMENTS}

Suppose that the finite element local space $V_{n}(\widehat{K})$ contains $P_{n}(\widehat{K})$, then according to Theorem 2.1 and Remark 2.2, the set of derivative superconvergent points can be characterized as

$$
\begin{array}{lll}
S P_{n}(\xi)=\{(\xi, \eta) \in \widehat{K} \mid & \frac{\partial \psi}{\partial \xi}(\xi, \eta)=0 & \left.\forall \psi \in \Psi_{n+1}(\widehat{K}) \backslash V_{n}(\widehat{K})\right\} \\
S P_{n}(\eta)=\{(\xi, \eta) \in \widehat{K} \mid & \frac{\partial \psi}{\partial \eta}(\xi, \eta)=0 & \left.\forall \psi \in \Psi_{n+1}(\widehat{K}) \backslash V_{n}(\widehat{K})\right\}
\end{array}
$$

Clearly, $S P_{n}(\xi)$ is the set of superconvergent points for derivatives in the $\xi$-direction and $S P_{n}(\eta)$ is the set of superconvergent points for derivatives in the $\eta$-direction.

Case 1. $P_{n+1}(\widehat{K}) \backslash\left\{\xi^{n+1}, \eta^{n+1}\right\} \subset V_{n}(\widehat{K}) \subset Q_{n}(\widehat{K})$, where $Q_{n}(\widehat{K})$ is the space of polynomials of degree not greater than $n$ in each variable on $\widehat{K}$. This includes the intermediate family, tensor-product elements and all possible choices in between.

$$
\Psi_{n+1}(\widehat{K}) \backslash V_{n}(\widehat{K})=\operatorname{Span}\left\{\phi_{n+1}(\xi), \phi_{n+1}(\eta)\right\} .
$$

Therefore

$$
\begin{aligned}
& S P_{n}(\xi)=\left\{(\xi, \eta) \in \widehat{K} \mid \quad \frac{\partial \phi_{n+1}}{\partial \xi}(\xi)=p_{n}(\xi)=0\right\}=\left\{\left(G_{i}^{(n)}, \eta\right), \quad i=1, \ldots, n\right\} \\
& S P_{n}(\eta)=\left\{(\xi, \eta) \in \widehat{K} \mid \quad \frac{\partial \phi_{n+1}}{\partial \eta}(\eta)=p_{n}(\eta)=0\right\}=\left\{\left(\xi, G_{i}^{(n)}\right), \quad i=1, \ldots, n\right\} .
\end{aligned}
$$

Here $G_{i}^{(n)}$ are zeros of the Legendre polynomial $p_{n}$, i.e., the Gaussian points of degree $n$.

Case 2. $V_{n}(\widehat{K})=S_{n}(\widehat{K})$, the serendipity family. This is a more interesting and also more complicated case where $\Psi_{n+1}(\widehat{K}) \cap V_{n}(\widehat{K})=\{0\}$ and hence 
$\Psi_{n+1}(\widehat{K}) \backslash V_{n}(\widehat{K})=\Psi_{n+1}(\widehat{K})$. The set of derivative superconvergent points are

$$
\begin{array}{lll}
S P_{n}(\xi)=\{(\xi, \eta) \in \widehat{K} \mid & \frac{\partial \psi}{\partial \xi}(\xi, \eta)=0 & \left.\forall \psi \in \Psi_{n+1}(\widehat{K})\right\} \\
S P_{n}(\eta)=\{(\xi, \eta) \in \widehat{K} \mid & \frac{\partial \psi}{\partial \eta}(\xi, \eta)=0 & \left.\forall \psi \in \Psi_{n+1}(\widehat{K})\right\}
\end{array}
$$

Therefore, we need to construct $\Psi_{n+1}(\widehat{K})$ explicitly by the Gram-Schmidt process. Toward this end, we introduce:

Theorem 4.1. For $k \geq 4,2 \leq i \leq k-1$, and $2 \leq j \leq k-2$,

$$
\int_{\widehat{K}} \nabla\left[\phi_{i}(\xi) \phi_{k+1-i}(\eta)\right] \cdot \nabla\left[\phi_{j}(\xi) \phi_{k-j}(\eta)\right] d \xi d \eta=0 .
$$

For $k-1>l \geq 4,2 \leq i \leq k-1$, and $2 \leq j \leq l-2$,

$$
\int_{\widehat{K}} \nabla\left[\phi_{i}(\xi) \phi_{k+1-i}(\eta)\right] \cdot \nabla\left[\phi_{j}(\xi) \phi_{l-j}(\eta)\right] d \xi d \eta=0 .
$$

For $k \geq 5,2 \leq i \leq k-1$, and $2 \leq j \leq k-3$,

$$
\begin{aligned}
& \int_{\widehat{K}} \nabla\left[\phi_{i}(\xi) \phi_{k+1-i}(\eta)\right] \cdot \nabla\left[\phi_{j}(\xi) \phi_{k-1-j}(\eta)\right] d \xi d \eta \\
= & \begin{cases}-\frac{1}{(2 i-1)(2 i-5)}\left\|p_{i-2}\right\|^{2}\left\|p_{k-i}\right\|^{2} & i=j+2, \\
-\frac{1}{(2 k-2 i+1)(2 k-2 i-3)}\left\|p_{i-1}\right\|^{2}\left\|p_{k-1-i}\right\|^{2} & i=j, \\
0 & \text { otherwise. }\end{cases}
\end{aligned}
$$

Proof. We shall utilize the fact that $\phi_{i}^{\prime}=p_{i-1}$ and the orthogonal properties $\left(p_{i}, p_{j}\right)=0$ if $i \neq j,\left(\phi_{i}, \phi_{j}\right)=0$ if $i>j+2$, and $\left(\phi_{i}, \phi_{i-1}\right)=0($ see $(3.2))$. We have

(1) When $k \geq 4,2 \leq i \leq k-1,2 \leq j \leq k-2$,

$$
\begin{aligned}
& \int_{\widehat{K}} \nabla\left[\phi_{i}(\xi) \phi_{k+1-i}(\eta)\right] \cdot \nabla\left[\phi_{j}(\xi) \phi_{k-j}(\eta)\right] d \xi d \eta \\
= & \left(p_{i-1}, p_{j-1}\right)\left(\phi_{k+1-i}, \phi_{k-j}\right)+\left(\phi_{i}, \phi_{j}\right)\left(p_{k-i}, p_{k-j-1}\right)=0,
\end{aligned}
$$

since for $i=j+1$,

$$
\left(p_{i-1}, p_{j-1}\right)=0=\left(\phi_{i}, \phi_{j}\right)
$$

for $i=j$,

$$
\left(\phi_{k+1-i}, \phi_{k-j}\right)=0=\left(p_{k-i}, p_{k-j-1}\right)
$$

and for $i<j$,

$$
\left(p_{i-1}, p_{j-1}\right)=0=\left(p_{k-i}, p_{k-j-1}\right) .
$$

(2) When $k-1>l \geq 4,2 \leq i \leq k-1$, and $2 \leq j \leq l-2$,

$$
\begin{aligned}
& \int_{\widehat{K}} \nabla\left[\phi_{i}(\xi) \phi_{k+1-i}(\eta)\right] \cdot \nabla\left[\phi_{j}(\xi) \phi_{l-j}(\eta)\right] d \xi d \eta \\
& =\left(p_{i-1}, p_{j-1}\right)\left(\phi_{k+1-i}, \phi_{l-j}\right)+\left(\phi_{i}, \phi_{j}\right)\left(p_{k-i}, p_{l-j-1}\right)=0,
\end{aligned}
$$

since for $i>j+2$,

$$
\left(p_{i-1}, p_{j-1}\right)=0=\left(\phi_{i}, \phi_{j}\right)
$$


for $i=j+1, j+2, i<j$,

$$
\left(p_{i-1}, p_{j-1}\right)=0=\left(p_{k-i}, p_{l-j-1}\right)
$$

and for $i=j$,

$$
\left(\phi_{k+1-i}, \phi_{l-j}\right)=0=\left(p_{k-i}, p_{l-j-1}\right) .
$$

(3) When $k \geq 5,2 \leq i \leq k-1$, and $2 \leq j \leq k-3$,

$$
\begin{aligned}
& \int_{\widehat{K}} \nabla\left[\phi_{i}(\xi) \phi_{k+1-i}(\eta)\right] \cdot \nabla\left[\phi_{j}(\xi) \phi_{k-1-j}(\eta)\right] d \xi d \eta \\
= & \left(p_{i-1}, p_{j-1}\right)\left(\phi_{k+1-i}, \phi_{k-1-j}\right)+\left(\phi_{i}, \phi_{j}\right)\left(p_{k-i}, p_{k-j-2}\right) .
\end{aligned}
$$

(a) If $i=j+1$ or $i>j+2$,

$$
\left(p_{i-1}, p_{j-1}\right)=0=\left(\phi_{i}, \phi_{j}\right)
$$

(b) If $i<j$,

$$
\left(p_{i-1}, p_{j-1}\right)=0=\left(p_{k-i}, p_{k-2-j}\right) .
$$

(c) If $i=j+2,\left(p_{i-1}, p_{j-1}\right)=0$ and

$$
\left(\phi_{i}, \phi_{j}\right)\left(p_{k-i}, p_{k-j-2}\right)=-\frac{1}{(2 i-1)(2 i-5)}\left\|p_{i-2}\right\|^{2}\left\|p_{k-i}\right\|^{2} .
$$

(d) If $i=j,\left(p_{k-i}, p_{k-j-2}\right)=0$ and

$$
\left(p_{i-1}, p_{j-1}\right)\left(\phi_{k+1-i}, \phi_{k-1-j}\right)=-\frac{1}{(2 k-2 i+1)(2 k-2 i-3)}\left\|p_{i-1}\right\|^{2}\left\|p_{k-1-i}\right\|^{2} .
$$

Here we have used the formula (3.2). The assertion follows by combining (a)(d).

Theorem 4.1 reveals the orthogonal properties (under the Laplace operator) among the interior basis functions of $P P_{n}(\widehat{K})$. (4.1) indicates that all interior basis functions of degrees $k+1$ are orthogonal to those of degrees $k(\geq 4),(4.2)$ implies that all interior basis functions of degrees $k+1(\geq 5)$ are orthogonal to those of degrees less than $k-2$, and (4.3) says that any interior basis function of degree $k+1$ is not orthogonal to at most two interior basis functions of degrees $k-1(\geq 4)$. These properties will dramatically simplify the Gram-Schmidt process in constructing $\Psi_{n+1}(\widehat{K})$.

The following is a list of $\Psi_{n+1}(\widehat{K})$ for $n=1,2,3,4,5,6$. Note that for $n \leq 2$, the serendipity family $S_{n}(\widehat{K})$ is the same as the intermediate family.

$$
\begin{aligned}
\Psi_{2}(\widehat{K})= & \operatorname{Span}\left\{\phi_{2}(\xi), \phi_{2}(\eta)\right\} \\
\Psi_{3}(\widehat{K})= & \operatorname{Span}\left\{\phi_{3}(\xi), \phi_{3}(\eta)\right\} \\
\Psi_{4}(\widehat{K})= & \operatorname{Span}\left\{\phi_{4}(\xi), p_{2}(\xi) p_{2}(\eta), \phi_{4}(\eta)\right\} \\
\Psi_{5}(\widehat{K})= & \operatorname{Span}\left\{\phi_{5}(\xi), \phi_{3}(\xi) p_{2}(\eta), p_{2}(\xi) \phi_{3}(\eta), \phi_{5}(\eta)\right\} \\
\Psi_{6}(\widehat{K})= & \operatorname{Span}\left\{\phi_{6}(\xi),\left(p_{4}(\xi)-\alpha p_{2}(\xi)\right) p_{2}(\eta), \phi_{3}(\xi) \phi_{3}(\eta)\right. \\
& \left.p_{2}(\xi)\left(p_{4}(\eta)-\alpha p_{2}(\eta)\right), \phi_{6}(\eta)\right\} \\
\Psi_{7}(\widehat{K})= & \operatorname{Span}\left\{\phi_{7}(\xi),\left(\phi_{5}(\xi)-\beta \phi_{3}(\xi)\right) p_{2}(\eta),\left(p_{4}(\xi)-\gamma p_{2}(\xi)\right) \phi_{3}(\eta)\right. \\
& \left.\phi_{3}(\xi)\left(p_{4}(\eta)-\gamma p_{2}(\eta)\right), p_{2}(\xi)\left(\phi_{5}(\eta)-\beta \phi_{3}(\eta)\right), \phi_{7}(\eta)\right\}
\end{aligned}
$$


Here

$$
\alpha=\frac{\left(p_{4}^{\prime}, p_{2}^{\prime}\right)}{2\left\|p_{2}^{\prime}\right\|^{2}}, \quad \beta=\frac{\left(\phi_{5}, \phi_{3}\right)\left\|p_{2}^{\prime}\right\|^{2}}{\left\|p_{2}\right\|^{4}+\left\|\phi_{3}\right\|^{2}\left\|p_{2}^{\prime}\right\|^{2}}, \quad \gamma=\frac{\left(p_{4}^{\prime}, p_{2}^{\prime}\right)\left\|\phi_{3}\right\|^{2}}{\left\|p_{2}\right\|^{4}+\left\|\phi_{3}\right\|^{2}\left\|p_{2}^{\prime}\right\|^{2}} .
$$

In order to simplify the notation, we denote

$$
\{f(\xi, \eta)=0\}=\{(\xi, \eta) \in \widehat{K} \mid \quad f(\xi, \eta)=0\} .
$$

Then, we have

$$
\begin{aligned}
S P_{1}(\xi) & =\left\{\phi_{2}^{\prime}(\xi)=0\right\}=\left\{p_{1}(\xi)=0\right\}=\{(0, \eta)\} \\
S P_{2}(\xi) & =\left\{\phi_{3}^{\prime}(\xi)=0\right\}=\left\{p_{2}(\xi)=0\right\}=\left\{\left( \pm \frac{1}{\sqrt{3}}, \eta\right)\right\} \\
S P_{3}(\xi) & =\left\{\phi_{4}^{\prime}(\xi)=0\right\} \cap\left\{p_{2}^{\prime}(\xi) p_{2}(\eta)=0\right\}=\left\{p_{3}(\xi)=0\right\} \cap\left\{\xi=0, p_{2}(\eta)=0\right\} \\
& =\left\{\xi=0, \pm \sqrt{\frac{3}{5}}\right\} \cap\left\{\xi=0, \eta= \pm \frac{1}{\sqrt{3}}\right\}=\left\{(0, \eta),\left( \pm \sqrt{\frac{3}{5}}, \pm \frac{1}{\sqrt{3}}\right)\right\} \\
S P_{4}(\xi) & =\left\{\phi_{5}^{\prime}(\xi)=0\right\} \cap\left\{\phi_{3}^{\prime}(\xi) p_{2}(\eta)=0\right\} \cap\left\{p_{2}^{\prime}(\xi) \phi_{3}(\eta)=0\right\} \\
& =\left\{p_{4}(\xi)=0\right\} \cap\left\{p_{2}(\xi)=0, \phi_{3}^{\prime}(\eta)=0\right\} \cap\left\{p_{2}^{\prime}(\xi)=0, \phi_{3}(\eta)=0\right\} \\
& =\left\{p_{4}(\xi)=0\right\} \cap\left\{\phi_{3}^{\prime}(\eta)=0\right\} \cap\left\{\phi_{3}(\eta)=0\right\}=\emptyset
\end{aligned}
$$

since $\left\{p_{4}(\xi)=0\right\} \cap\left\{p_{2}(\xi)=0\right\}=\emptyset$, and $\left\{p_{4}(\xi)=0\right\} \cap\left\{p_{2}^{\prime}(\xi)=0\right\}=\emptyset$.

$$
\begin{aligned}
S P_{5}(\xi)= & \left\{\phi_{6}^{\prime}(\xi)=0\right\} \cap\left\{\left(p_{4}^{\prime}(\xi)-\alpha p_{2}^{\prime}(\xi)\right) p_{2}(\eta)=0\right\} \\
& \cap\left\{\phi_{3}^{\prime}(\xi) \phi_{3}(\eta)=0\right\} \cap\left\{p_{2}^{\prime}(\xi)\left(p_{4}(\eta)-\alpha p_{2}(\eta)\right)=0\right\} \\
= & \left\{p_{5}(\xi)=0\right\} \cap\left\{\xi=0, p_{2}(\eta)=0\right\} \cap\left\{\phi_{3}(\eta)=0\right\} \\
& \cap\left\{\xi=0, p_{4}(\eta)-\alpha p_{2}(\eta)=0\right\} \\
= & \left\{p_{5}(\xi)=0\right\} \cap\{\xi=0\} \cap\left\{\phi_{3}(\eta)=0\right\} \cap\{\xi=0\} \\
= & \{\xi=0\} \cap\left\{\phi_{3}(\eta)=0\right\}=\{(0,0),(0, \pm 1)\} ;
\end{aligned}
$$

since

$$
\begin{gathered}
\left\{p_{5}(\xi)=0\right\} \cap\left\{p_{4}^{\prime}(\xi)-\alpha p_{2}^{\prime}(\xi)=0\right\}=\{\xi=0\}, \\
\left\{p_{5}(\xi)=0\right\} \cap\left\{p_{2}(\xi)=0\right\}=\emptyset, \\
\left\{\phi_{3}(\eta)=0\right\} \cap\left\{p_{2}(\eta)=0\right\}=\emptyset, \\
\left\{\phi_{3}(\eta)=0\right\} \cap\left\{p_{4}(\eta)-\alpha p_{2}(\eta)=0\right\}=\emptyset . \\
S P_{6}(\xi) \subset\left\{\phi_{7}^{\prime}(\xi)=0\right\} \cap\left\{\left(\phi_{5}^{\prime}(\xi)-\beta \phi_{3}^{\prime}(\xi)\right) p_{2}(\eta)=0\right\} \\
\cap\left\{\phi_{3}^{\prime}(\xi)\left(p_{4}(\eta)-\gamma p_{2}(\eta)\right)=0\right\} \\
=\left\{p_{6}(\xi)=0\right\} \cap\left\{p_{4}(\xi)-\beta p_{2}(\xi)=0, p_{2}(\eta)=0\right\} \\
\cap\left\{p_{2}(\xi)=0, p_{4}(\eta)-\gamma p_{2}(\eta)=0\right\} \\
=\left\{p_{6}(\xi)=0\right\} \cap\left\{p_{2}(\eta)=0\right\} \cap\left\{p_{4}(\eta)-\gamma p_{2}(\eta)=0\right\}=\emptyset ;
\end{gathered}
$$


since

$$
\begin{array}{r}
\left\{p_{6}(\xi)=0\right\} \cap\left\{p_{4}(\xi)-\beta p_{2}(\xi)=0\right\}=\emptyset \\
\left\{p_{6}(\xi)=0\right\} \cap\left\{p_{2}(\xi)=0\right\}=\emptyset .
\end{array}
$$

The superconvergent points for the other derivative can be obtained similarly. Summing up, we conclude that:

1. For any finite element space contained in the tensor-product space that contains the intermediate family, all superconvergent points for Poisson's equation under the rectangular mesh are along Gaussian lines.

2. For the serendipity family of order $n=3$, the superconvergent points (in the reference element) are along the central line $\xi=0$ and at four points $\left( \pm \sqrt{\frac{3}{5}}, \pm \sqrt{\frac{1}{3}}\right)$ for $\frac{\partial u}{\partial x}$; and along the central line $\eta=0$ and at four points $\left( \pm \sqrt{\frac{1}{3}}, \pm \sqrt{\frac{3}{5}}\right)$ for $\frac{\partial u}{\partial y}$.

For the serendipity family of order $n=4,6$ (and likely any even order $n \geq 4$ ), there is no superconvergent point.

For the serendipity family of order $n=5$ (and likely any odd order $n \geq 5$ ), there are three superconvergent points: the element center and mid-points of two parallel edges.

3. Any finite element space that contains $P_{n}(\widehat{K})$ will have at least all superconvergent points of the serendipity family.

Remark 4.1. All results in the computer-based proof of [1] for Poisson's equation under the rectangular mesh are justified. But, the results here are more general in the sense that they include all possible choices for the finite element space between the intermediate family and the tensor-product space and all possible finite element spaces that contain $P_{n}(\widehat{K})$, the complete polynomial space of degree $n$.

Remark 4.2. For the serendipity family, the most interesting case is $n=3$, in which we have theoretically justified the computer-based discovery in [1]. For odd $n \geq 5$, all three superconvergent points have been predicted theoretically in [5]. The contribution here is to verify that they are the only superconvergent points.

Remark 4.3. The superconvergence results in this work are restricted to the Poisson equation while the "symmetry" theory is for more general second-order elliptic equations. However, it is possible to extend the findings here to some other equations. This belief is encouraged by the following fact: While original superconvergent points in [3] were restricted to the Poisson equation, the result has been generalized to the equation (see [7], Chapter 6)

$$
-\nabla(\alpha(x) \nabla u)-\sum_{i=1}^{n} \frac{\partial}{\partial x_{i}}\left(a_{i}(x) u\right)+a(x) u=f .
$$

\section{Derivative SuperConvergent POINTS FOR BRICK ELEMENTS}

In this section, superconvergent points for the three dimensional Poisson equation under local brick elements will be investigated. This development is based on the observation in Remark 2.3, therefore a parallel result of Theorem 2.1 in 3-D is our starting point here. The discussion will be brief. Now the reference element is 
$\widehat{K}=[-1,1]^{3}$, and

$$
\begin{array}{r}
P P_{n}(\widehat{K})=\operatorname{Span}\left\{1, \phi_{k}(\xi), \phi_{k}(\eta), \phi_{k}(\zeta), k=2,3, \ldots, n ; \phi_{i}(\xi) \phi_{j}(\eta), \phi_{i}(\eta) \phi_{j}(\zeta),\right. \\
\left.\phi_{i}(\zeta) \phi_{j}(\xi), i+j \leq n, i, j \geq 2 ; \phi_{i}(\xi) \phi_{j}(\eta) \phi_{k}(\zeta), i+j+k \leq n, i, j, k \geq 2\right\}
\end{array}
$$

Case 1. $P_{n+1}(\widehat{K}) \backslash\left\{\xi^{n+1}, \eta^{n+1}, \zeta^{n+1}\right\} \subset V_{n}(\widehat{K}) \subset Q_{n}(\widehat{K})$. This includes the three dimensional intermediate family and tensor-product elements and all possible choices in between.

$$
\Psi_{n+1}(\widehat{K}) \backslash V_{n}(\widehat{K})=\operatorname{Span}\left\{\phi_{n+1}(\xi), \phi_{n+1}(\eta), \phi_{n+1}(\zeta)\right\}
$$

Therefore

$$
\begin{aligned}
& S P_{n}(\xi)=\left\{(\xi, \eta, \zeta) \in \widehat{K} \mid \quad \frac{\partial \phi_{n+1}}{\partial \xi}(\xi)=p_{n}(\xi)=0\right\}=\left\{\left(G_{i}^{(n)}, \eta, \zeta\right), \quad i=1, \ldots, n\right\} \\
& S P_{n}(\eta)=\left\{(\xi, \eta, \zeta) \in \widehat{K} \mid \quad \frac{\partial \phi_{n+1}}{\partial \eta}(\eta)=p_{n}(\eta)=0\right\}=\left\{\left(\xi, G_{i}^{(n)}, \zeta\right), \quad i=1, \ldots, n\right\} \\
& S P_{n}(\zeta)=\left\{(\xi, \eta, \zeta) \in \widehat{K} \mid \quad \frac{\partial \phi_{n+1}}{\partial \zeta}(\zeta)=p_{n}(\zeta)=0\right\}=\left\{\left(\xi, \eta, G_{i}^{(n)}\right), \quad i=1, \ldots, n\right\}
\end{aligned}
$$

Hence derivative superconvergent points are on the Gaussian planes.

Case 2. $V_{n}(\widehat{K})$ is the 3-D serendipity family. Now we have,

$$
\begin{array}{llll}
S P_{n}(\xi)=\{(\xi, \eta, \zeta) \in \widehat{K} \mid & \frac{\partial \psi}{\partial \xi}(\xi, \eta, \zeta)=0 & \left.\forall \psi \in \Psi_{n+1}(\widehat{K})\right\} \\
S P_{n}(\eta)=\{(\xi, \eta, \zeta) \in \widehat{K} \mid & \frac{\partial \psi}{\partial \eta}(\xi, \eta, \zeta)=0 & \left.\forall \psi \in \Psi_{n+1}(\widehat{K})\right\} \\
S P_{n}(\zeta)=\{(\xi, \eta, \zeta) \in \widehat{K} \mid & \frac{\partial \psi}{\partial \zeta}(\xi, \eta, \zeta)=0 & \left.\forall \psi \in \Psi_{n+1}(\widehat{K})\right\}
\end{array}
$$

Here

$$
\begin{aligned}
\Psi_{2}(\widehat{K})= & \operatorname{Span}\left\{\phi_{2}(\xi), \phi_{2}(\eta), \phi_{2}(\zeta)\right\} \\
\Psi_{3}(\widehat{K})= & \operatorname{Span}\left\{\phi_{3}(\xi), \phi_{3}(\eta), \phi_{3}(\zeta)\right\} \\
\Psi_{4}(\widehat{K})= & \operatorname{Span}\left\{\phi_{4}(\xi), \phi_{4}(\eta), \phi_{4}(\zeta), p_{2}(\xi) p_{2}(\eta), p_{2}(\eta) p_{2}(\zeta), p_{2}(\zeta) p_{2}(\xi)\right\} \\
\Psi_{5}(\widehat{K})= & \operatorname{Span}\left\{\phi_{5}(\xi), \phi_{5}(\eta), \phi_{5}(\zeta), \phi_{3}(\xi) p_{2}(\eta), p_{2}(\xi) \phi_{3}(\eta)\right. \\
& \left.\phi_{3}(\eta) p_{2}(\zeta), p_{2}(\eta) \phi_{3}(\zeta), \phi_{3}(\zeta) p_{2}(\xi), p_{2}(\zeta) \phi_{3}(\xi)\right\} \\
\Psi_{6}(\widehat{K})=\operatorname{Span}\left\{\phi_{6}(\xi), \phi_{6}(\eta), \phi_{6}(\zeta)\right. & \quad\left(p_{4}(\xi)-\alpha p_{2}(\xi)\right) p_{2}(\eta), \phi_{3}(\xi) \phi_{3}(\eta), p_{2}(\xi)\left(p_{4}(\eta)-\alpha p_{2}(\eta)\right) \\
& \left(p_{4}(\eta)-\alpha p_{2}(\eta)\right) p_{2}(\zeta), \phi_{3}(\eta) \phi_{3}(\zeta), p_{2}(\eta)\left(p_{4}(\zeta)-\alpha p_{2}(\zeta)\right) \\
& \left.\left(p_{4}(\zeta)-\alpha p_{2}(\zeta)\right) p_{2}(\xi), \phi_{3}(\zeta) \phi_{3}(\xi), p_{2}(\zeta)\left(p_{4}(\xi)-\alpha p_{2}(\xi)\right)\right\}
\end{aligned}
$$


Therefore,

$$
\begin{aligned}
S P_{1}(\xi)= & \left\{p_{1}(\xi)=0\right\}=\{(0, \eta, \zeta)\} \\
S P_{2}(\xi)= & \left\{p_{2}(\xi)=0\right\}=\left\{\left( \pm \frac{1}{\sqrt{3}}, \eta, \zeta\right)\right\} \\
S P_{3}(\xi)= & \left\{p_{3}(\xi)=0\right\} \cap\left\{\xi=0, p_{2}(\eta)=0\right\} \cap\left\{\xi=0, p_{2}(\zeta)=0\right\} \\
= & \left\{(0, \eta, \zeta),\left( \pm \sqrt{\frac{3}{5}}, \pm \frac{1}{\sqrt{3}}, \pm \frac{1}{\sqrt{3}}\right)\right\} \\
S P_{4}(\xi)= & \left\{p_{4}(\xi)=0\right\} \cap\left\{p_{2}(\xi)=0, p_{2}(\eta)=0\right\} \cap\left\{\xi=0, \phi_{3}(\eta)=0\right\} \\
& \cap\left\{p_{2}(\xi)=0, p_{2}(\zeta)=0\right\} \cap\left\{\xi=0, \phi_{3}(\zeta)=0\right\}=\emptyset ; \\
S P_{5}(\xi)= & \left\{p_{5}(\xi)=0\right\} \cap\left\{\left(p_{4}^{\prime}(\xi)-\alpha p_{2}^{\prime}(\xi)\right)=0, p_{2}(\eta)=0\right\} \\
& \cap\left\{p_{2}(\xi)=0, \phi_{3}(\eta)=0\right\} \cap\left\{\xi=0,\left(p_{4}(\eta)-\alpha p_{2}(\eta)\right)=0\right\} \\
& \cap\left\{\left(p_{4}^{\prime}(\xi)-\alpha p_{2}^{\prime}(\xi)\right)=0, p_{2}(\zeta)=0\right\} \cap\left\{p_{2}(\xi)=0, \phi_{3}(\zeta)=0\right\} \\
& \cap\left\{\xi=0,\left(p_{4}(\zeta)-\alpha p_{2}(\zeta)\right)=0\right\} \\
= & \{(0,0,0),(0, \pm 1,0),(0,0, \pm 1),(0, \pm 1, \pm 1)\} .
\end{aligned}
$$

Superconvergent points for the other two partial derivatives can be obtained similarly. In summary, we have shown:

1. For any finite element space contained in the tensor-product space that contains the intermediate family, all superconvergent points for Poisson's equation under the brick mesh are on Gaussian planes.

2. For the serendipity family of order $n=3$, the superconvergent points (in the reference element) are on the central plane $\xi=0$ and at eight points

$$
\left( \pm \sqrt{\frac{3}{5}}, \pm \sqrt{\frac{1}{3}}, \pm \sqrt{\frac{1}{3}}\right)
$$

for $\frac{\partial u}{\partial x}$. Similar results hold for the other two partial derivatives.

For the serendipity family of order $n=4$ (and likely any even order $n \geq 4$ ), there is no superconvergent point.

For the serendipity family of order $n=5$ (and likely any odd order $n \geq 5$ ), there are nine superconvergent points: element center, mid-points of four parallel edges and centers of four associated faces. Note that the element center is shared by all three partial derivatives, and each face center is shared by two partial derivatives.

Any finite element space that contains $P_{n}(\widehat{K})$ will have at least all superconvergent points of the serendipity family.

\section{ACKNOWLEDGMENTS}

The author would like to thank Professor Lars B. Wahlbin for his encouragement and helpful discussions on the present work. The author would also like to thank the referee for some valuable suggestions.

\section{REFERENCES}

1. I. Babuška, T. Strouboulis, C.S. Upadhyay, and S.K. Gangaraj, Computer-based proof of the existence of superconvergence points in the finite element method; superconvergence of the derivatives in finite element solutions of Laplace's, Poisson's, and the elasticity equations, Numerical Methods for Partial Differential Equations 12 (1996), 347-392. MR 97c:65160 
2. C.-M. Chen, Superconvergent points of Galerkin's method for two-point boundary value problems (in Chinese), Numer. Math. J. Chinese Univ. 1 (1979), 73-79.

3. J. Douglas, Jr., T. Dupont, and M.F. Wheeler, An $L^{\infty}$ estimate and a superconvergence result for a Galerkin method for elliptic equations based on tensor products of piecewise polynomials, RAIRO 8 (1974), 61-66. MR 50:11812

4. N.N. Lebedev, Special Functions and Their Applications, Dover, New York, 1972. MR 50:2568

5. A.H. Schatz, I.H. Sloan, and L.B. Wahlbin, Superconvergence in finite element methods and meshes which are symmetric with respect to a point, SIAM Journal on Numerical Analysis 33(2) (1996), 505-521. CMP 96:12

6. B. Szabó and I. Babuška, Finite Element Analysis, John Wiley \& Sons, New York, 1991. MR 93f: 73001

7. L.B. Wahlbin, Superconvergence in Galerkin Finite Element Methods, Lecture Notes in Mathematics, Vol. 1605, Springer, Berlin, 1995. CMP 97:09

Department of Mathematics, Texas Tech University, Lubbock, Texas 79409

E-mail address: zhang@ttmath.ttu.edu 